\title{
An Approach for the Construction of Systems That Self-Generate Chaotic Solitons
}

\author{
Baoying Chen \\ Department of Applied Mathematics, Guangdong University of Technology, Guangzhou, China \\ Email: sunnycby@126.com
}

Received April 28, 2012; revised May 28, 2012; accepted June 5, 2012

\begin{abstract}
This paper proposes a method for constructing partial differential equation (PDE) systems with chaotic solitons by using truncated normal forms of an ordinary differential equation (ODE). The construction is based mainly on the fact that the existence of a soliton in a PDE system is equal to that of a homoclinic orbit in a related ODE system, and that chaos of Ši'lnikov homoclinic type in the ODE imply that the soliton in the PDE changes its profile chaotically along propagation direction. It is guaranteed that the constructed systems can self-generate chaotic solitons without any external perturbation but with constrained wave velocities in a rigorously mathematical sense.
\end{abstract}

Keywords: Chaotic Solitons; Partial Differential Equation; Homoclinic Orbit

\section{Introduction}

Chaotic solitons have been a subject of many theoretical papers over the last decades of years. They have been experimentally observed in several physical systems [14]. Despite this large amount of effort, many key issues remain open. The central question addressed in this paper is: How can one construct a partial differential equation (PDE) system that self-generates a chaotic solitary-wave pulse that exists in a rigorous sense? There are few rigorous results that addresses this question. In most cases, rigorous results for the generation of chaotic solitons are obtained through perturbations to a known system, e.g., Schrödinger equation and Ginzburg-Landau equation [58], that can generate solitons. Unfortunately, this method is not regular since, in practice, it can be applied only to particular examples. Another rigorous approach is that of C. L. Zheng [9]. In this approach one must find variable separation solutions to a generalized $(2+1)$-dimensional Kotewege de-Vries equation by using Bäcklund transformations [10]. The approach can be in nature categorized to perturbation method. A third approach, by Wu et al. in [3] and Ricketts et al. in [4], is an experimental scheme. The former described a related system that deliberately provokes inherently nonlinear dynamics to produce chaotic soliton oscillations. The latter showed that the cancellation does more than simply allow the faithful propagation of digital pulses: in fact, it can be used as part of electrical oscillator to produce pulses in the first place. Both of these systems are purely electronic; their relative ease of manufacture gives them many advantages over the "photonic" devices, involving light waves, that currently dominate soliton research. As defined in [8], a chaotic soliton means that the soliton changes its profile chaotically in time or along propagation direction. From the view of mathematics, a soliton of a PDE system corresponds to a special solution of the PDE, or equally, a soliton corresponds to a homoclinic orbit in a related ordinary differential equation (ODE). Furthermore, if such an orbit is of Shilnikov's type [11], that is, some Shilnikov's inequalities are satisfied, then the ODE system definitely has chaos of Shilnikov's type. It is not difficult to imagine that the corresponding soliton in the original PDE system should be a chaotic soliton since its profile impossibly behaves regularly in time. Such a relation between some solutions of ODE and PDE systems is the basis of our constructing PDE systems that self-generate chaotic solitons. On the other hand, from an ODE system with homoclinic orbits, one may construct many PDE systems such that they can self-generate solitons corresponding to the homoclinic orbits in the ODE system. Therefore, the construction must be treated carefully so that the resulting systems via the construction are as desired.

In this paper, we propose a method for constructing a PDE system self-generating chaotic solitons. The construction is based on the truncated normal forms of an autonomous ODE and on the relationship between ODEs and PDEs when solitary waves are under consideration. The constructed system can self-generate a chaotic solitary-wave pulse without any external perturbation. Moreover, the existence of the solitary-wave pulse in the PDE 
system is ensured by that of one homoclinic orbit of an ODE system whereas its chaotic behavior (i.e., the soliton changes its profile chaotically along propagation direction) results from the chaos property of the ODE system which is guaranteed by Ši'lnikov's homoclinic theorem [11].

\section{A Candidate Class of ODE Systems and Their Principle Homoclinic Orbits}

Since finding a soliton in a PDE system can be changed into proving the existence of a homoclinic orbit in a related ODE system, in this section we will introduce a class of autonomous ODE systems as candidates for the construction of PDE systems that can self-generate chaotic solitons. The introduction of the candidate ODE systems is based on amplitude equations which are derived from normal form theory. Then, we discuss the existence of homoclinic orbits in the ODE systems. We do not prove the existence of the homoclinic orbit, and instead obtain some so-called principle homoclinic orbits by applying perturbation theory. Furthermore, we show that such homoclinic orbits are of Ši'lnikov's type, implying that the ODE systems have chaos of Ši'lnikov's type.

We begin by a general theory about the standard form of nonlinear ODE systems. In the analysis of the stability of static states of autonomous nonlinear ODE systems, linear theory produces normal modes that vary like $t^{k} e^{s t}$, where $t$ is time, $k$ is an integer, and $s=\sigma \pm \omega i$ is a root of the characteristic value equation of the linear theory. Consider an autonomous nonlinear ODE system having a polycritical state in which $\sigma=\omega=0$ for $l$ roots and $\sigma=0$ with $\omega \neq 0$ for $m$ pairs of complex conjugate roots. Suppose that, for this state, $\sigma$ is bounded away from zero for all the other roots of the characteristic equation. By using normal form theory, we can obtain amplitude equations of the form:

$$
x^{\prime}=J_{\mu} x+g_{\mu}(x)
$$

where $x=\left(x_{1}, x_{2}, \cdots, x_{d}\right)^{T}$ with $d=l+2 m$ is the amplitude vector, $g_{\mu}$ is a strictly nonlinear vector-valued function, and $\mu=\left(\mu_{0}, \cdots, \mu_{n-1}\right)$ is a set of parameters. We restrict ourselves to cases where the number of parameters in the characteristic polynomial for $J_{\mu}$ is equal to $n$ (called criticality). In this case, the characteristic equation (called the critical polynomial) for matrix $J_{\mu}$ may be expressed as

$$
\operatorname{det}\left(J_{\mu}-s I\right)=s^{n}+\sum_{k=0}^{n-1} \mu_{k} s^{k} \equiv P_{n}(\mu ; s)=0
$$

We are interested here in the leading-order terms in $g_{\mu}$, e.g., for $n=2$, they are of order $x^{2}$. These leading terms in $g_{\mu}$ are dictated by $J_{0}$. For the critical condi- tion, $\mu=(v, \lambda)=0$ in the case of $n=2$, the characteristic equation has zero as a double root. We refer to this case as $\xi^{2}$ when $J_{0}$ is an irreducible $2 \times 2$ matrix. Similarly, we have other $\xi^{n}$ (see [12]). In addition, when $J_{\mu}$ is in Jordan-Arnold form [12,13], we rewrite Equation (1) as

$$
\left\{\begin{array}{l}
x_{1}^{\prime}=x_{2}, x_{2}^{\prime}=x_{3}, \cdots, x_{n-1}^{\prime}=x_{n} \\
x_{n}^{\prime}+\sum_{l=1}^{n} \mu_{l-1} x_{l}=\sum_{m=1}^{n} \sum_{l=1}^{m} \alpha_{l m} x_{m+1-l} x_{l} \equiv g\left(x_{k}\right)
\end{array}\right.
$$

where the $\alpha_{l m}$ are constants which can be analytically calculated [14].

Let $\varepsilon$ be a small positive number and introduce the scaled variables

$$
\tau=\varepsilon t, X_{k}(\tau)=\varepsilon^{1-n-k} X_{k}(t), v_{i}=\varepsilon^{i-n} \mu_{i}
$$

where $i=1,2, \cdots, n-1$. If we arrange matters so that the $v_{i}$ are of order unity, the values of $\mu_{i}$ are near to the critical condition $\mu_{i}=0$. That is, we position the system in that part of parameter space where, for $i=1,2, \cdots, n-1$, $\mu_{i}=O\left(\varepsilon^{n-i}\right)$. Then Equation (3) becomes

$$
\left\{\begin{array}{l}
X_{1}^{\prime}=X_{2}, X_{2}^{\prime}=X_{3}, \cdots, X_{n-1}^{\prime}=X_{n}, \\
X_{n}^{\prime}+\sum_{l=1}^{n} v_{l-1} X_{l}=\varepsilon^{-2 n} g\left(\varepsilon^{n+k-1} X_{k}\right) .
\end{array}\right.
$$

If we keep only the leading order terms in $g$, we may reduce Equation (5) to

$$
\frac{\mathrm{d}^{n} X}{\mathrm{~d} \tau^{n}}+\sum_{l=0}^{n-1} v_{l} \frac{\mathrm{d}^{l} X}{\mathrm{~d} \tau^{l}}=k_{1} X^{2},
$$

where $X=X_{1}, k_{1}=\alpha_{11}$ and we are ignoring terms $O(\varepsilon)$. Once we are within the region of parameter space delimited by $\mu_{I}=O\left(\varepsilon^{n-i}\right)$, we may use Equation (6) as the amplitude equation for the configuration $\xi^{n}$, for any $n$, with the error of order $\varepsilon$.

We are interested especially in the case of $n=3$. In this case, we have the following truncated normal form as the amplitude equation for $\xi^{3}$ (see [13,14]):

$$
x_{t}^{\prime \prime \prime}=-\left(\eta-k_{4} x\right) x_{t}^{\prime \prime}-\left(v-k_{3} x-k_{2} x^{\prime}\right) x^{\prime}-\left(\lambda-k_{1} x\right) x
$$

where $k_{i}(1 \leq i \leq 4)$ and $v$ are constants. Equation (7) has two singular points $x_{*}=0$ and $x_{*}=\lambda / k_{1}$. We look for solutions of the form $x_{*}+\xi$. If $|\xi|$ is small, $\xi \propto e^{\text {st }}$, and we obtain

$$
s^{3}+\left(\eta-k_{4} X_{*}\right) s^{2}+\left(v-k_{3} X_{*}\right) s+\lambda-2 k_{1} X_{*}=0
$$

The parameter $\eta$ is a measure of the dissipation, and assumed to be positive. A change in the sign of $\lambda$ essentially interchanges the rules of the two singular points, so we need consider only $\lambda<0$. When $|v| \ll|\eta|$ and $|\lambda| \ll|\eta|$, we can obtain some principle homoclinic orbits by applying perturbation theory [12], e.g., for fixed $k_{1}=-1, k_{2}=k_{3}=0, k_{4}=1$, there is a principle homo- 
clinic orbit for $\xi^{3}$ when $\eta$ is small and the Ši'lnikov condition $v^{\prime}<-\lambda^{\prime}-2$ and $\lambda^{\prime}<-1$ is satisfied with $\lambda^{\prime}=\lambda / \eta^{3}, v^{\prime}=v / \eta^{2} \quad$ (refer Figure 1). The region for existence of principle homoclinic orbits constitutes a cusp in shape, which is given by

$$
4\left(1 / 3-v^{\prime}\right)^{3}=3\left(3 \lambda^{\prime}-v^{\prime}+2 / 9\right)^{2}
$$

and the cusp has a vertex at $v^{\prime}=1 / 3, \quad \lambda^{\prime}=1 / 27$ (see Figure 1). Besides these principle homoclinic orbits, there are many other homoclinic orbits in the problem (see [15]). Such a homoclinic orbit will imply the existence of chaos of Ši'lnikov type for an ODE system and ensure the existence of a chaotic soliton for a related PDE (see details in the next section).

\section{Constructing a PDE System That Self-Generates a Chaotic Soliton}

A particular example is the asymptotic normal form for $\xi^{3}$ which is of the form:

$$
X_{t}^{\prime \prime \prime}+X_{t}^{\prime \prime}+b X_{t}^{\prime}-a X+X^{2}=0
$$

where $X$ has been scaled to give a convenient coefficient to $X^{2}$. Based on such an ODE and inspired by the relation between ODE and PDE when the travelling waves are considered, we construct the following model:

$$
\tilde{b} \frac{\partial u}{\partial t}=\frac{\partial^{3} u}{\partial r^{3}}+\frac{\partial^{2} u}{\partial r^{2}}-a u+u^{2}
$$

where $a$ and $\tilde{b}$ are positive constants. Assume that

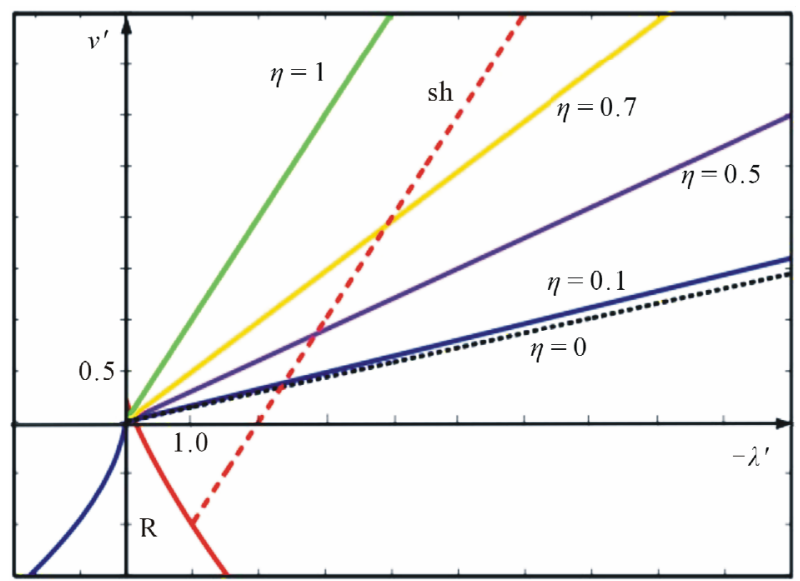

Figure 1. The principle homoclinic conditions for the case $k_{1}$ $=-1, k_{2}=k_{3}=0, k_{4}=1$ of $\xi^{3}$. The different curves are labelled by the values of $\eta$ to which they correspond. The dotted curve corresponds to the location of the principle homoclinic orbit of the asymptotic form for $\xi^{3}$ (see Equation (1)). Outside the cusp-shaped region $R$ given by $4\left(1 / 3-v^{\prime}\right)^{3}=3\left(3 \lambda^{\prime}-v^{\prime}+2 / 9\right)^{2}$, the origin is a saddle-focus. To the right of the red curve marked by $\mathrm{Sh}$, the Ši'Inikov condition $v^{\prime}<-\lambda^{\prime}-2$ and $\lambda^{\prime}<-1$ satisfied.
Equation (10) has travelling waves of the standard form $u(r, t) \equiv U(\varsigma)$, where $\varsigma=r-c t$ and $c$ represents travelling wave speed (assume $c>0$ ). Then, $U$ satisfies Equation (9) with $b=\tilde{b} c$. Similarly, we can construct the classical Korteweg-de Vries (KdV) equation which has been applied to the optical fibers communication field if we set $\eta=0, \lambda=0, k_{1}=k_{2}=0$ and $k_{3}=-1, k_{4}=0$, based on the ODE (8). Now, we seek a solitary wave solution, that is, we find the solution of the following equation $U_{\varsigma \varsigma \varsigma}^{\prime \prime \prime}+U_{\varsigma \varsigma}^{\prime \prime}+b U_{\varsigma}^{\prime}-a U+U^{2}=0$ subjective to the constrain $U \stackrel{\varsigma \varsigma}{\rightarrow} a, \quad U_{\varsigma}^{\prime} \rightarrow 0, \quad U_{\varsigma \varsigma}^{\prime \prime} \rightarrow 0$ as $\varsigma \rightarrow \infty$, where the subscript stands for derivatives of function $U$ with respect to the argument $\varsigma$. For this, we rewrite Equation (9) into an ODE

$$
\left\{\begin{array}{l}
\frac{\mathrm{d} x}{\mathrm{~d} \tau}=y \\
\frac{\mathrm{d} y}{\mathrm{~d} \tau}=z \\
\frac{\mathrm{d} z}{\mathrm{~d} \tau}=a x-x^{2}-b y-z
\end{array}\right.
$$

with the condition $x \rightarrow a, y \rightarrow 0$ and $z \rightarrow 0$ as $\tau \rightarrow \infty$, where $b=\tilde{b} c$. Note that finding a soliton solution of Equation (10) satisfying the condition is currently changed into finding a homoclinic orbit of Equation (11) based at the equilibrium point $(a, 0,0)$.

Fourth-order Rounge-Kutta method is used to solve the system (11) with time step size equal to 0.01 , the absolute and relative error equal to $10^{-6}$. Numerical simulation shows that the system (11) is chaotic when $a=3.5$ and $b=2$, as shown in Figure 2. Now, let us fix $a=3.5$. The numerical simulation also shows that the existence interval of chaos reaches $b \in[1.88,2.03]$. Thus, we can determine the range of the solitary-wave velocity for fixed $\tilde{b}$.

We can verify analytically that Equation (10) indeed has a Ši'lnikov homoclinic orbit and further chaos of Ši'lnikov type from a theorem of Ši'lnikov [11]. In fact, we have shown the existence of a homoclinic orbit (e.g., the principle homoclinic orbit) of Ši'lnikov type. Figure 3 shows the numerical soliton of Equation (10) according to the homoclinic orbit.

In addition, the characteristic polynomial of Jacobian matrix of Equation (10) evaluated at $(a, 0,0)$ is $\sigma^{3}+\sigma^{2}+\alpha \sigma+a=0$ which has three roots: one negative $\sigma_{1}=-1.3833$ and a conjugate pair of complex $\sigma_{2,3}=0.1916 \pm 1.5791 i$ with the positive real part for $a=3.5$ and $\alpha=2$. A rigorously theoretical proof for the existence of chaos of Ši'lnikov type may be referred to a standard treatment in [16]. A chaotic soliton of Equation (10) with parameter $a=3.5$ and $\tilde{b}=-5.2$ is shown in Figure 4 (the numerical simulation is performed for $10^{4}$ iterations with a time step of 0.001). 


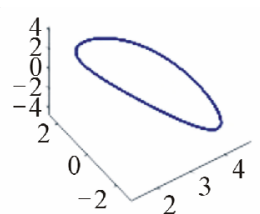

(a)

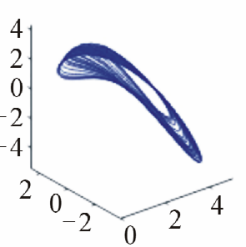

(d)

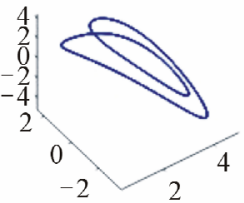

(b)

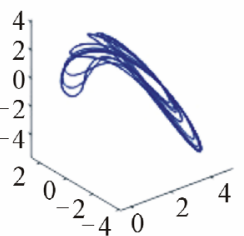

(e)

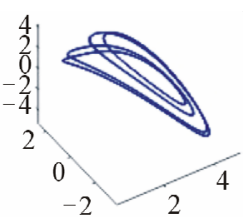

(c)

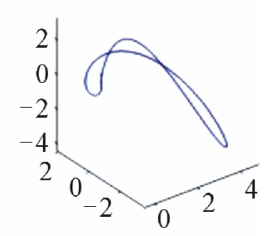

(f)
Figure 2. Evolution of the attractor of the asymptotic form for $\xi^{3}$ described by Equation (9) or (11) when the parameter $b$ is (a) 2.5 ; (b) 2.1 ; (c) 2.05 ; (d) 2.0 ; (e) 1.88 ; (f) 1.419 for fixed $a=3.5$. Here (a), (b), (c) illustrate the period-doubling cascade leading to the numerical strange attractor shown in (d) and (e). The principle homoclinic orbit is represented in (f): it is unstable. Transients are suppressed.

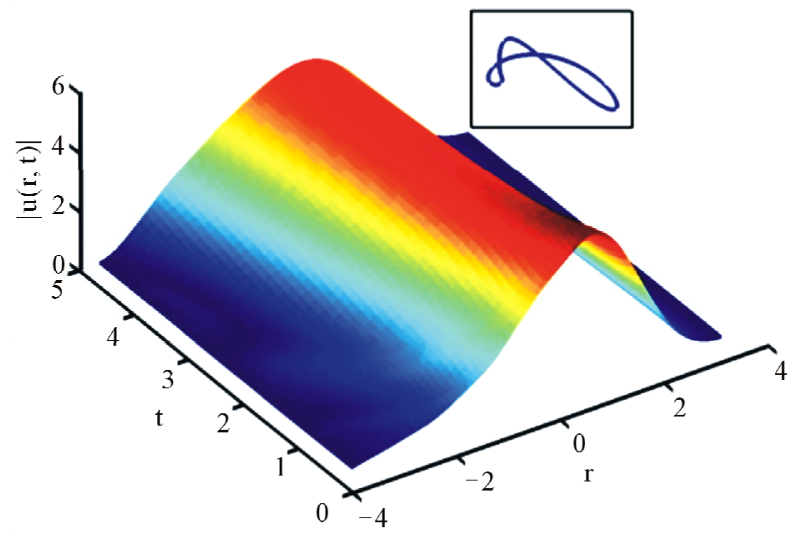

Figure 3. The numerical soliton, where parameter $a=3.5$, $\tilde{b}=-3.7$.

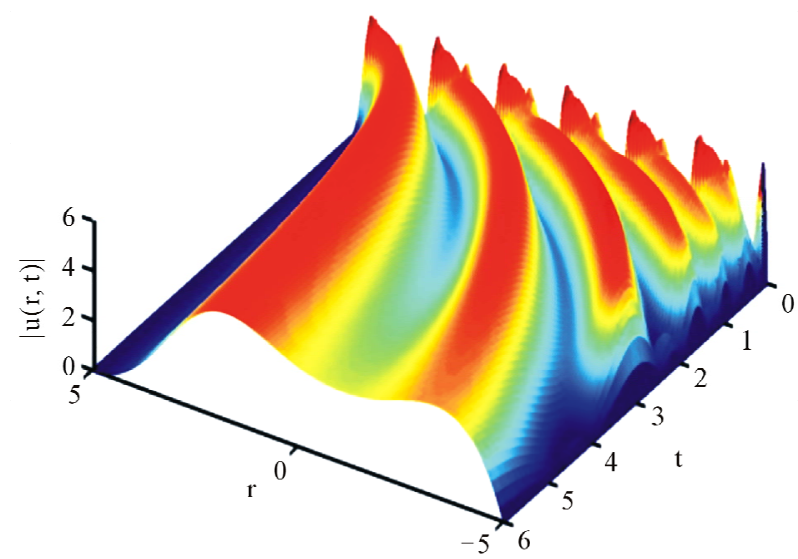

Figure 4. A numerical chaotic soliton, where parameter $a=$ 3.5 and $\tilde{b}=-5.2$.

\section{Conclusion}

Based on normal form theory of ODEs, we have proposed a general method of constructing systems that selfgenerate chaotic solitons. By adjusting some system parameters in truncated normal forms of ODEs (e.g., Equation (8)), one can construct many systems with chaotic solitons. Note that the constructed system (10) is conservative. It seems the first example that self-generates chaotic solitons.

\section{REFERENCES}

[1] T. H. Lee, "Electrical Solitons Come of Age," Nature, Vol. 440, 2006, pp. 36-37. doi:10.1038/440036a

[2] A. Argyris, et al., "Chaos-Based Communications at High Bit Rates Using Commercial Fibre-Optic Links,” Nature, Vol. 438, 2005, pp. 343-346. doi:10.1038/nature04275

[3] M. Z. Wu, B. A. Kalinikos and C. E. Patton, "Self-Generation of Chaotic Solitary Spin Wave Pulses in Magnetic Film Active Feedback Rings," Physical Review Letters, Vol. 95, No. 23, 2005, 237202. doi:10.1103/PhysRevLett.95.237202

[4] D. S. Riketts, X. Li and D. Ham, "Electrical Soliton Oscillator," IEEE Transactions on Microwave Theory and Techniques, Vol. 54, No. 1, 2006, pp. 373-382. doi:10.1109/TMTT.2005.861652

[5] S. V. Dmitriev, et al., "Chaotic Character of Two-Soliton Collisions in the Weakly Perturbed Nonlinear Schrödinger Equation,” Physical Review E, Vol. 66, No. 4, 2002, 046609. doi:10.1103/PhysRevE.66.046609

[6] S. V. Dmitriev and T. Shigenari, "Short-Lived Two- Soliton Bound States in Weakly Perturbed Nonlinear Schrödinger Equation,” Chaos, Vol. 12, No. 2, 2002, pp. 324-332. doi:10.1063/1.1476951

[7] F. Benvenuto, et al., "Manifestations of Classical and Quantum Chaos in Nonlinear Wave Propagation,” Physical Review A, Vol. 44, No. 6, 1991, R3423. doi:10.1103/PhysRevA.44.R3423

[8] N. Akhmediev, et al., "Pulsating Solitons, Chaotic Solitons, Period Doubling, and Pulse Coexistence in ModeLocking Lasers: Complex Ginzburg-Landau Equation Approach,” Physical Review E, Vol. 63, No. 5, 2001, Article ID: 056602. doi:10.1103/PhysRevE.63.056602

[9] C. L. Zheng, "Coherent Soliton Structures with Chaotic and Fractal Behaviors in a Generalized (2+1)-Dimensional Korteweg de-Vries System," Chinese Journal of Physics, Vol. 41, 2003, pp. 442-456.

[10] S. P. Novikov and A. P. Veselov, "Two-Dimensional Schrödinger Operator: Inverse Scattering Transform and Evolutional Equations,” Physica D: Nonlinear Phenomena, Vol. 18, No. 1-3, 1986, pp. 267-273. doi:10.1016/0167-2789(86)90187-9

[11] F. C. P. Silva, “Ši'l'nikov Theorem-A Tutorial,” IEEE Transactions on Circuits and Systems, Vol. 40, 1993, pp. 675-682.

[12] V. I. Arnold, "Lectures in Bifurcation in Versal Fami- 
lies,” Russian Mathematical Surveys, Vol. 27, No. 5, 1972, pp. 54-123. doi:10.1070/RM1972v027n05ABEH001385

[13] A. Arneodo, et al., “Asymptotic Chaos," Physica D: Nonlinear Phenomena, Vol. 14, No. 3, 1985, pp. 327-347. doi:10.1016/0167-2789(85)90093-4

[14] P. H. Coullet and E. A. Spiegel, "Amplitude Equations for Systems with Competing Instabilities,” SIAM Journal on Applied Mathematics, Vol. 43, No. 4, 1983, pp. 776-
821. doi:10.1137/0143052

[15] A. Arneodo, et al., "Oscillators with Chaotic Behavior: An Illustration of a Theorem by Shilnikov,” Journal of Statistical Physics, Vol. 27, 1982, pp. 171-182. doi:10.1007/BF01011745

[16] S. Wiggins, “Global Bifurcation and Chaos,” SpringerVerlag, New York, 1988.

doi:10.1007/978-1-4612-1042-9 